\title{
A matched-pair analysis of clinical outcomes after intracavitary cesium-131 brachytherapy versus stereotactic radiosurgery for resected brain metastases
}

\author{
*Diana A. Julie, MD, MPH, ${ }^{1}$ Stefanie P. Lazow, MD, ${ }^{2}$ Daniel B. Vanderbilt, MD, PhD, ${ }^{1}$ \\ Shoshana Taube, MD, ${ }^{1}$ Menachem Z. Yondorf, MD, ${ }^{1}$ Albert Sabbas, PhD, ${ }^{1}$ Susan Pannullo, MD, ${ }^{3}$ \\ Theodore H. Schwartz, MD, ${ }^{3}$ and A. Gabriella Wernicke, MD, MSc ${ }^{1,3}$ \\ ${ }^{1}$ Stich Radiation Oncology, Weill Cornell Medical College/NewYork-Presbyterian Hospital, New York, New York; ${ }^{2}$ Department \\ of Surgery, Beth Israel Deaconess Medical Center/Harvard Medical School, Boston, Massachusetts; and 'Department of \\ Neurosurgery, Brain and Spine Center, Weill Cornell Medical College/NewYork-Presbyterian Hospital, New York, New York
}

OBJECTIVE Adjuvant radiation therapy (RT), such as cesium-131 (Cs-131) brachytherapy or stereotactic radiosurgery (SRS), reduces local recurrence (LR) of brain metastases (BM). However, SRS is less efficacious for large cavities, and the delay between surgery and SRS may permit tumor repopulation. Cs-131 has demonstrated improved local control, with reduced radiation necrosis (RN) compared to SRS. This study represents the first comparison of outcomes between Cs-131 brachytherapy and SRS for resected BM.

METHODS Patients with BM treated with Cs-131 and SRS following gross-total resection were retrospectively identified. Thirty patients who underwent Cs-131 brachytherapy were compared to 60 controls who received SRS. Controls were selected from a larger cohort to match the patients treated with Cs-131 in a 2:1 ratio according to tumor size, histology, performance status, and recursive partitioning analysis class. Overall survival (OS), LR, regional recurrence, distant recurrence (DR), and $\mathrm{RN}$ were compared.

RESULTS With a median follow-up of 17.5 months for Cs-131-treated and 13.0 months for SRS-treated patients, the LR rate was significantly lower with brachytherapy; $10 \%$ for the Cs-131 cohort compared to $28.3 \%$ for SRS patients (OR 0.281, $95 \% \mathrm{Cl} 0.082-0.949 ; p=0.049$ ). Rates of regional recurrence, $\mathrm{DR}$, and OS did not differ significantly between the two cohorts. Kaplan-Meier analysis with log-rank testing showed a significantly higher likelihood of freedom from LR $(p=0.027)$ as well as DR ( $p=0.018$ ) after Cs-131 compared to SRS treatment $(p=0.027)$, but no difference in likelihood of OS $(p=0.093)$. Six (10.0\%) patients who underwent SRS experienced RN compared to $1(3.3 \%)$ patient who received Cs-131 ( $p=0.417)$.

CONCLUSIONS Postresection patients with BM treated with Cs-131 brachytherapy were more likely to achieve local control compared to SRS-treated patients. This study provides preliminary evidence of the potential of Cs-131 to reduce LR following gross-total resection of single BM, with minimal toxicity, and suggests the need for a prospective study to address this question.

https://thejns.org/doi/abs/10.3171/2020.3.JNS193419

KEYWORDS brachytherapy; brain metastases; cesium-131; Cs-131; stereotactic radiosurgery; SRS; radiation necrosis; oncology

$\mathrm{R}$ ESECTION of brain metastases (BM) results in local recurrence (LR) in nearly half of patients, underscoring the need for additional therapy. ${ }^{1}$ When administered adjuvantly, whole-brain radiation therapy (WBRT) reduces death from neurological causes, as well as LR, from $46 \%$ to $10 \%{ }^{1-4}$ However, WBRT is associated with neurocognitive deficits and reduced quality of life, establishing the need for more localized radiation therapy (RT)..$^{5-7}$ Two potential localized RT techniques are stereotactic radiosurgery (SRS) and intracavitary brachytherapy.

ABBREVIATIONS BM = brain metastases; DR = distant recurrence; DRFS = DR-free survival; ECOG = Eastern Cooperative Oncology Group; GTR = gross-total resection; KPS = Karnofsky Performance Scale; LC = local control; LR = local recurrence; LRFS = LR-free survival; OS = overall survival; RN = radiation necrosis; RPA = recursive partitioning analysis; $R R=$ regional recurrence; RRFS = RR-free survival; $R T$ = radiation therapy; $S R S$ = stereotactic radiosurgery; WBRT = whole-brain radiation therapy. SUBMITTED December 19, 2019. ACCEPTED March 10, 2020.

INCLUDE WHEN CITING Published online May 15, 2020; DOI: 10.3171/2020.3.JNS193419.

${ }^{*}$ D.A.J. and S.P.L. share first authorship of this work. 
SRS achieves similar rates of postoperative local control (LC) to WBRT, with fewer neurocognitive sequelae. ${ }^{1,5,6,8}$ However, SRS may be less efficacious for large tumors (> $2.5 \mathrm{~cm})$ and resected cavity volumes $\left(>15 \mathrm{~cm}^{3}\right){ }^{9-11}$ Additionally, the necessary delay between resection and SRS may permit tumor repopulation, reducing LC.

Another localized RT option is intracavitary brachytherapy, in which radioactive seeds are implanted directly within the resection cavity. Numerous studies have reported that brachytherapy using iodine-125 (I-125) achieves comparable LC to adjuvant WBRT or SRS. However, I-125 intracavitary brachytherapy has drawn criticism due to high rates (up to $40 \%$ ) of radiation necrosis $(\mathrm{RN}) .{ }^{12} \mathrm{To}$ minimize toxicity, our institution uses a novel FDA-approved isotope, cesium-131 (Cs-131), with a substantially shorter half-life than I-125 (9.7 vs 59.4 days). This allows > $90 \%$ of the dose to be delivered within 1 month, in contrast to I-125, for which only one-third of the dose is deposited over this time. Cs-131 achieves improved dosimetry, is less sensitive to temporal resection cavity changes, and results in less RN. ${ }^{13-15}$ It has been demonstrated prospectively that Cs-131 offers excellent LC, with low rates of RN..$^{15}$ To our knowledge, no studies have directly compared the efficacy of adjuvant SRS and brachytherapy performed with Cs131. We aimed to compare clinical outcomes and risk of $\mathrm{RN}$ for these focal RT options.

\section{Methods \\ Patient Selection}

With IRB approval, consecutive patients treated with resection and brachytherapy at our institution between 2002 and 2014 were retrospectively selected for study inclusion. Patients undergoing Cs-131 brachytherapy were part of an IRB-approved prospective trial. ${ }^{15}$ Surgery was indicated to relieve mass effect, reduce symptoms, obtain samples for diagnostic pathology studies, or for tumor diameter $>2.5 \mathrm{~cm}$. Eastern Cooperative Oncology Group (ECOG)/Zubrod Performance Status 0-2 and minimum expected survival of 6 months were required. Exclusion criteria included tumor proximity to the chiasm or brainstem, small cell carcinoma pathology, and pregnancy or refusal to use birth control. From this group of patients who underwent Cs-131 implantation, 30 patients were selected for inclusion after fulfilling these additional eligibility criteria: gross-total resection (GTR), no prior WBRT, no prior surgical or radiation treatment of the lesion treated in this study, and follow-up of at least 1 month. GTR was defined on postoperative MRI as lack of residual contrast-enhancing nodularity or mass, as determined by the reviewing neuroradiologist.

For comparison, a group of 60 patients who received adjuvant SRS during the same time period, and who also met the aforementioned inclusion criteria, were retrospectively selected. These controls were randomly selected from an existing institutional database of patients with resected BM to match the patients who underwent Cs-131 treatment in a 2:1 ratio. To create clinically comparable cohorts, patients were matched according to preoperative tumor maximum dimension, histology, RPA (recursive partitioning analysis) class, and ECOG performance sta- tus. Tumor size was matched within $\pm 5 \mathrm{~mm}$. With regard to histology, pathological diagnosis was matched, but further subcategories such as hormonal status or molecular markers were not matched. ECOG status and RPA class were defined at the time of resection. ${ }^{2,3}$ When multiple SRS-treated patients matched, 1 patient was randomly selected. Matching was conducted by a study investigator, and to avoid bias, was performed with de-identified patient information, and blinded to patient outcomes or to any clinical variables beyond those used for matching. Although patients were not matched with regard to sex, race, Karnofsky Performance Scale (KPS) status, active systemic disease, tumor location, presence of additional $\mathrm{BM}$, surgical complications, or prior intracranial treatments, these variables did not differ significantly between the two cohorts, as shown in Table 1. Patients were eligible for inclusion even if additional BM were present at the time of resection; these synchronous lesions were treated with SRS. Patients who underwent SRS in addition to resection were still included if there was evidence of tumor recurrence in the interval between resection and SRS, as an intention-to-treat analysis.

\section{Brachytherapy Dosimetry}

After resection, 10-cm suture-stranded Cs-131 seeds (IsoRay) with a median activity of $3.8 \mathrm{mCi}$ (IQR 3.5-4.0 $\mathrm{mCi}$ ) were permanently implanted within the tumor bed, with $0.5-\mathrm{cm}$ interseed spacing. Strands were placed tangentially within the resection cavity, with interstrand separation of 7-10 mm, and secured with Surgicel and Tisseel. ${ }^{14}$ A median of 14.5 seeds (IQR 8.25-20 seeds) were placed per cavity. A dose of 80 Gy was prescribed to a 5-mm depth from the cavity surface. Implant volume was determined based on preoperative tumor volume and our institutional physics nomogram, with real-time adjustment for postresection intracavitary volume. Implant dose distribution was assessed with CT imaging within 24-48 hours postoperatively.

\section{SRS Dosimetry}

SRS was performed using linear accelerator (LINAC) Varian technology (Novalis, ExacTrac), prescribed to the $80 \%$ isodose line. Prescriptions ranged from 18 to $33 \mathrm{~Gy}$ (median $24 \mathrm{~Gy}$ ) in 1-5 fractions, to a 2-mm margin surrounding the resection cavity. The SRS dose was at the discretion of the treating radiation oncologist. For BM treated at our institution, commonly used SRS regimens include 18-20 Gy in 1 fraction, 24-33 Gy in 3 fractions, or 25-30 Gy in 5 fractions. Dose and fractionation is determined by target volume-with larger volumes receiving a lower dose or greater fractionation - and by proximity to organs at risk or ability to achieve dose constraints. Our institutional practice is to respect the American Association of Physicists in Medicine (AAPM) Task Group 101 constraints. ${ }^{16}$

\section{Demographic and Baseline Data}

Preoperative tumor size was determined based on the greatest dimension on MRI. Patients were characterized as having active systemic disease if there was radiograph- 
TABLE 1. Characteristics in 90 patients with BM treated using either Cs-131 brachytherapy or SRS

\begin{tabular}{|c|c|c|c|}
\hline Characteristic & Cs-131* & SRS $^{*}$ & p Value $†$ \\
\hline No. of patients & 30 & 60 & \\
\hline Median age in yrs at op (IQR) & $62.0(53.3-71.8)$ & $65.0(57.3-70.0)$ & 0.622 \\
\hline Sex & & & 0.551 \\
\hline Male & $16(53.3)$ & $28(46.7)$ & \\
\hline Female & $14(46.7)$ & $32(53.3)$ & \\
\hline Race & & & 0.658 \\
\hline White & $23(76.7)$ & $42(70.0)$ & \\
\hline Black & $2(6.7)$ & $8(13.3)$ & \\
\hline Asian & $1(3.3)$ & $5(8.3)$ & \\
\hline Hispanic & $3(10.0)$ & $3(5.0)$ & \\
\hline Other or declined & $1(3.3)$ & $2(3.3)$ & \\
\hline KPS score & & & 0.877 \\
\hline$\geq 90$ & $9(30.0)$ & $17(28.3)$ & \\
\hline 80 & $9(30.0)$ & $23(38.3)$ & \\
\hline 70 & $11(36.7)$ & $18(30.0)$ & \\
\hline$<70$ & $1(3.3)$ & $2(3.3)$ & \\
\hline Active systemic disease & $20(66.7)$ & $43(71.7)$ & 0.626 \\
\hline Tumor histology & & & 1.000 \\
\hline Non-small cell lung cancer & $18(60.0)$ & $36(60.0)$ & \\
\hline Breast & $3(10.0)$ & $6(10.0)$ & \\
\hline Colorectal & $3(10.0)$ & $6(10.0)$ & \\
\hline Gastric \& esophageal & $2(6.7)$ & $4(6.7)$ & \\
\hline Endometrial & $1(3.3)$ & $2(3.3)$ & \\
\hline Melanoma & $1(3.3)$ & $2(3.3)$ & \\
\hline Oropharynx & $1(3.3)$ & $2(3.3)$ & \\
\hline Renal & $1(3.3)$ & $2(3.3)$ & \\
\hline Tumor location & & & 0.420 \\
\hline Cerebellar & $7(23.3)$ & $14(23.3)$ & \\
\hline Frontal & $8(26.7)$ & $24(40.0)$ & \\
\hline Occipital & $2(6.7)$ & $3(5.0)$ & \\
\hline Parietal & $10(33.3)$ & $11(18.3)$ & \\
\hline Temporal & $2(6.7)$ & $8(13.3)$ & \\
\hline Frontoparietal & $1(3.3)$ & $0(0)$ & \\
\hline Presence of additional BM at time of op & $10(33.3)$ & $32(53.3)$ & 0.073 \\
\hline Previous SRS to other BM & $1(3.3)$ & $3(5.0)$ & 1.000 \\
\hline Previous resection \pm brachytherapy to other BM & $2(6.7)$ & $3(5.0)$ & 1.000 \\
\hline
\end{tabular}

Unless otherwise indicated, values are expressed as the number of patients (\%).

* All percentages are calculated within the respective Cs-131 or SRS group.

$\dagger p$ values are calculated from Mann-Whitney U-test, chi-square test, or Fisher's exact test, as appropriate.

$\ddagger$ Variable used for matching.

ic evidence of untreated primary disease, radiographic progression of primary or metastatic sites, or receipt of systemic chemotherapy in the month preceding resection.

\section{Clinical Outcome Measures}

Clinical outcomes investigated include overall survival (OS); recurrence (LR, regional recurrence [RR], and distant recurrence [DR]); need for salvage therapy; and RN. LR was defined on surveillance MRI as new or increased nodular contrast enhancement $\leq 5 \mathrm{~mm}$ from the resection cavity; RR was defined as new or increased nodularity or contrast enhancement $>5 \mathrm{~mm}$ to $\leq 20 \mathrm{~mm}$ from the cavity or adjacent leptomeningeal disease; and DR was defined as new or increased contrast enhancement or nodularity elsewhere in the brain. ${ }^{17}$ Recurrence was evaluated with postoperative MRI and clinical follow-up, assessing for new or progressive neurological deficits. MRI included the following sequences: T1-weighted, FLAIR, T2-weighted, gradient echo, and diffusion-weighted imaging. Duration of OS and time to recurrence were measured from 
TABLE 2. Radiographic findings and clinical outcomes in 90 patients with BM treated using either Cs-131 brachytherapy or SRS

\begin{tabular}{|c|c|c|c|}
\hline Characteristic & Cs-131* & SRS* & p Value $†$ \\
\hline No. of patients & 30 & 60 & \\
\hline Median preop tumor size in cm (IQR) $\ddagger$ & $2.7(2.1-3.2)$ & $2.8(2.3-3.4)$ & 0.387 \\
\hline Median follow-up duration in mos (IQR) & $17.5(7.8-57.8)$ & $13.0(5.3-24.0)$ & 0.078 \\
\hline Surgery complications & & & 0.646 \\
\hline Infection & $1(3.3)$ & $1(1.7)$ & \\
\hline Seizures & $1(3.3)$ & $3(5.0)$ & \\
\hline Hematoma & $0(0)$ & $1(1.7)$ & \\
\hline LR & $3(10)$ & $17(28.3)$ & 0.049 \\
\hline RR & $5(16.7)$ & $6(10)$ & 0.363 \\
\hline DR & $14(46.7)$ & $40(66.7)$ & 0.068 \\
\hline OS & $20(66.7)$ & $34(56.7)$ & 0.361 \\
\hline RN & $1(3.3)$ & $6(10.0)$ & 0.417 \\
\hline Additional SRS to same metastasis & $2(6.7)$ & $1(1.7)$ & 0.257 \\
\hline $\begin{array}{l}\text { Additional resection } \pm \text { brachytherapy implantation of } \\
\text { same metastasis }\end{array}$ & $0(0)$ & $6(10.0)$ & 0.173 \\
\hline Additional SRS to different metastasis & $11(36.7)$ & $36(60.0)$ & 0.037 \\
\hline $\begin{array}{l}\text { Any additional resection } \pm \text { brachytherapy of different } \\
\text { metastasis }\end{array}$ & $6(20.0)$ & $9(15.0)$ & 0.549 \\
\hline Additional WBRT & $4(13.3)$ & $9(15.0)$ & 1.000 \\
\hline \multicolumn{4}{|c|}{$\begin{array}{l}\text { Unless otherwise indicated, values are expressed as the number of patients (\%). } \\
\text { * All percentages are calculated within the respective Cs-131 or SRS population. } \\
\dagger p \text { values are calculated from Mann-Whitney U-test, chi-square test, Fisher's exact test, and Kaplan-Meier log-rank } \\
\text { test analyses, as appropriate. } \\
\ddagger \text { Variable used for matching. }\end{array}$} \\
\hline
\end{tabular}

the date of resection. Salvage therapy was defined as any subsequent surgical or RT intervention for management of intracranial disease. The Radiation Therapy Oncology Group (RTOG) scale was used to evaluate RN. ${ }^{18}$

\section{Statistical Analysis}

Statistical analysis was performed with SPSS (version 22; IBM Corp.) and Prism (version 8.3.1; GraphPad Software, Inc.), and a 2 -sided $\mathrm{p}$ value $<0.05$ was considered significant. Nonparametric Mann-Whitney U-test, chisquare test, and Fisher's exact test were used to compare demographic variables and clinical outcomes between the cohorts. Kaplan-Meier analysis with log-rank (MantelCox) testing was performed for comparison of OS and freedom from recurrence. Recurrences were censored at time of last follow-up or death.

\section{Results}

Demographic and baseline clinical information is presented in Table 1 . The median age at surgery was 62.0 years (IQR 53.3-71.8 years) and 65.0 years (IQR 57.370.0 years) in the Cs-131 and SRS groups, respectively. There were $20(66.7 \%)$ patients in the Cs-131 group and $43(71.7 \%)$ patients in the SRS group who had active systemic disease $(p=0.626)$. As demonstrated in Table 1 , the Cs-131 and SRS cohorts did not differ significantly in any key baseline characteristics, including age, sex, race, KPS score, presence of systemic disease, tumor location, or prior treatments.

\section{Clinical Outcomes}

Surgical complications in the SRS cohort included 3 postoperative seizures (5.0\%) and 1 fluid collection (1.7\%). In the Cs-131 cohort, postoperative seizure occurred in 1 patient $(3.3 \%)$ and infection occurred in 1 patient $(3.3 \%)$. Overall, rates of surgical complications were low, and did not differ significantly between the cohorts. The median follow-up was 17.5 months (IQR 7.8-57.8 months) for patients who received Cs-131 brachytherapy and 13.0 months (IQR 5.3-24.0 months) for those who underwent SRS. There was no significant difference in follow-up duration for the two cohorts $(p=0.078)$. The median preoperative tumor size did not differ significantly between the cohorts: $2.7 \mathrm{~cm}$ (IQR 2.1-3.2 cm) for Cs-131 versus $2.8 \mathrm{~cm}$ (IQR 2.3-3.4 cm) for SRS ( $\mathrm{p}=0.387$ ).

Rates of OS, recurrence, RN, and salvage therapy are presented in Table 2. Kaplan-Meier analysis with log-rank testing comparing LR, DR, and OS between cohorts is shown in Fig. 1.

Patients treated with Cs-131 had a significantly lower LR rate; $3(10 \%)$ compared to 17 (28.3\%) in the SRS group (OR 0.281, 95\% CI 0.082-0.949; $\mathrm{p}=0.049$ ). Kaplan-Meier analysis with log-rank testing demonstrated significantly increased LR-free survival (LRFS) in the Cs-131 com- 

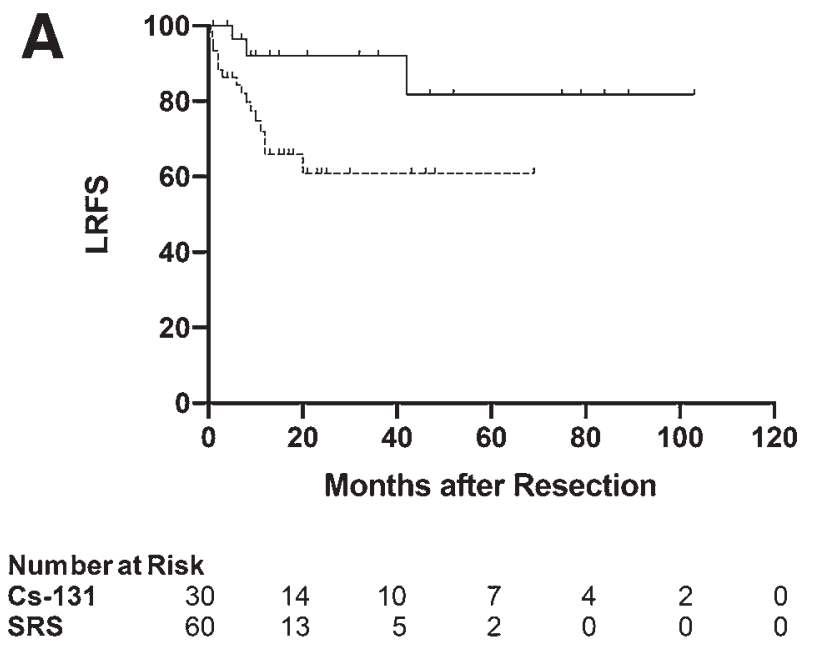

$\begin{array}{lrrrrrrr}\text { Cs-131 } & 30 & 14 & 10 & 7 & 4 & 2 & 0 \\ \text { SRS } & 60 & 13 & 5 & 2 & 0 & 0 & 0\end{array}$
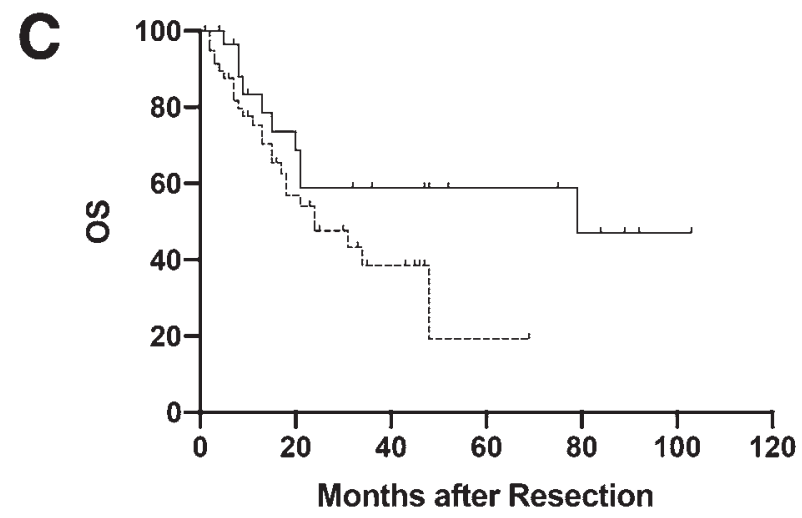

B

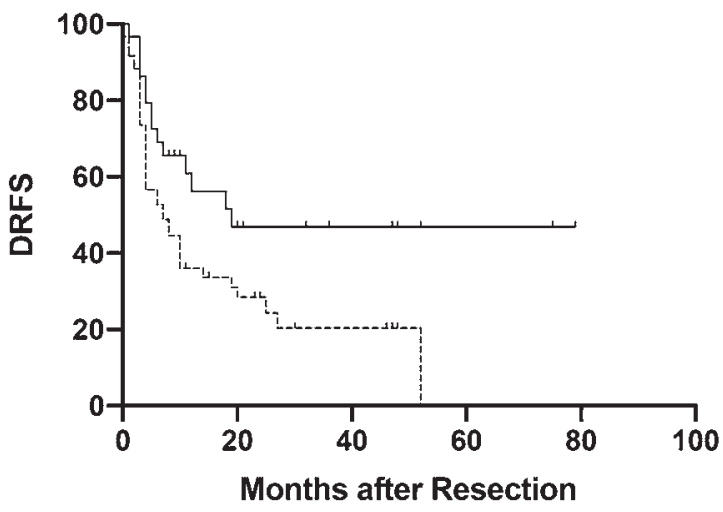

Number at Risk

$\begin{array}{lllllll}\text { Cs-131 } & 30 & 10 & 7 & 4 & 1 & 0 \\ \text { SRS } & 60 & 12 & 5 & 1 & 0 & 0\end{array}$

Number at Risk

$\begin{array}{lrrrrrrr}\text { Cs-131 } & 30 & 15 & 11 & 8 & 5 & 2 & 0 \\ \text { SRS } & 60 & 22 & 8 & 2 & 0 & 0 & 0\end{array}$

FIG. 1. A: Kaplan-Meier survival curves illustrating LRFS in the Cs-131 brachytherapy group $(n=30)$ versus the SRS group $(n=$ 60). B: Kaplan-Meier survival curves illustrating DRFS in the Cs-131 brachytherapy group $(n=30)$ versus the SRS group $(n=60)$. C: Kaplan-Meier survival curves illustrating OS in the Cs-131 brachytherapy group $(n=30)$ versus the SRS group $(n=60)$.

pared to the SRS cohorts $(p=0.027$; Fig. $1 \mathrm{~A})$. There was a significantly longer delay between surgery and SRS in patients experiencing LR (median 60.0 days [IQR 38.0-68.0 days]) and those not experiencing LR (median 34.0 days [IQR 26.0-44.0 days], $\mathrm{p}<0.001$ ). No significant association was observed between preoperative tumor size and risk of LR in either the Cs-131 $(\mathrm{p}=0.695)$ or SRS $(\mathrm{p}=$ $0.863)$ cohort, separately or combined $(p=0.627)$.

RR occurred in 5 (16.7\%) Cs-131 patients and $6(10.0 \%)$ SRS patients, with no significant difference between the groups (OR 1.8, 95\% CI 0.545-6.252; $\mathrm{p}=0.363$ ). There was no significant difference in RR-free survival (RRFS) between the cohorts on log-rank testing $(\mathrm{p}=0.721)$. DR occurred in 14 (46.7\%) Cs-131 and 40 (66.7\%) SRS patients, and this was not significantly different between the groups (OR $0.438,95 \%$ CI $0.185-1.113 ; p=0.068$ ). On Kaplan-Meier analysis with log-rank testing, the Cs-131 cohort had a significantly higher likelihood of DR-free survival (DRFS) compared to the SRS cohort $(\mathrm{p}=0.018$;
Fig. 1B). Twenty (66.7\%) Cs-131 patients and 34 (56.7\%) SRS patients were alive at last follow-up (OR 1.529, 95\% CI $0.600-4.026 ; \mathrm{p}=0.361$ ), with no significant difference between the groups. Likewise, there was no significant difference in OS between the groups on log-rank testing $(p=0.093$; Fig. 1C).

One (3.3\%) Cs-131 patient experienced RN, whereas $6(10.0 \%)$ SRS patients developed this complication $(\mathrm{p}=$ 0.417 ), at a median of 9 months (range 2-25 months) following RT. Salvage WBRT was administered in $9(15.0 \%)$ SRS patients, compared to $4(13.3 \%)$ Cs-131 patients ( $\mathrm{p}=$ 1.000). One (1.7\%) SRS patient required additional SRS to the same metastatic lesion, whereas $6(10.0 \%)$ SRS patients required additional resection of the same metastatic site, with subsequent brachytherapy implantation. Two (6.7\%) Cs-131 patients required salvage SRS to the same lesion (Table 2). Between the cohorts, there was no significant difference in overall salvage therapy rates to the same $\mathrm{BM}(\mathrm{p}=0.714)$. 


\section{Discussion}

Resection of BM alone yields unacceptably high LR, approaching $50 \%$, so adjuvant RT is the standard of care. ${ }^{1,719}$ SRS and intracavitary brachytherapy are two localized adjuvant RT modalities used to reduce LR. Numerous studies demonstrate that SRS and brachytherapy result in comparable clinical outcomes to WBRT, which has been the traditional adjuvant RT method. . $, 5,8,12$ SRS has emerged as an alternative to WBRT due to high LC rates, coupled with reduced toxicity, especially neurocognitive defects. However, two factors adversely affect LC following postoperative SRS: 1) large tumor size ( $>2.5 \mathrm{~cm})$ and resection cavity volume $\left(>15 \mathrm{~cm}^{3}\right)$; and 2) delay between resection and SRS, allowing for tumor repopulation., ${ }^{5,8-11,20,21}$ Intracavitary brachytherapy has been demonstrated in numerous studies to confer LC of $80 \%-100 \%$, comparable to WBRT, but has received criticism on account of high rates of RN with the use of I-125. ${ }^{12}$ To address these toxicity concerns, our institution uses the novel FDA-cleared isotope Cs-131. This isotope has a shorter half-life than I-125 (9.7 days vs 59.4 days), resulting in a shorter duration of dose delivery, such that radiation dosimetry is less affected by resection cavity shrinkage..$^{14}$ A previous study reported that Cs-131 results in reduced $\mathrm{RN}$ compared to I-125. ${ }^{15}$

This study represents the first direct comparison of clinical outcomes between SRS and Cs-131 brachytherapy after GTR of a BM. We report significantly higher LR after SRS compared to Cs-131, $28 \%$ compared to $10 \%$. Similarly, SRS conferred significantly reduced LRFS. Additionally, $10 \%$ of patients who underwent SRS developed $\mathrm{RN}$, compared to only $3 \%$ of patients who received Cs131. Our results suggest that Cs-131 confers superior LC to SRS, without increased RN risk.

Our LC rate for postoperative SRS is very consistent with rates reported in the literature (approximately 70\%), especially for larger tumor volumes; our finding of increased LC with Cs-131 is novel..$^{5,6,19,21}$ A comparative study of SRS and I-125 brachytherapy found no significant differences in median OS (8.1 months with SRS vs 8.0 months with brachytherapy), LC (93.6\% vs $96.7 \%)$, or distant control (42.4\% vs $46.4 \%)$ between the two modalities. ${ }^{22}$

Our finding of increased LC with Cs-131 brachytherapy probably reflects several benefits of intracavitary brachytherapy over SRS, including radiobiological advantages, enhanced ability to treat larger tumor cavities, and reduced interval between resection and RT. Because RT is most effective at treating smaller tumor volumes, if there is a delay in adjuvant SRS and tumor repopulation occurs, the risk of treatment failure increases. Whereas SRS is administered in either a single fraction or a few daily fractions, Cs-131 delivers radiation at a low dose rate. Use of low-dose-rate radiation allows for increased repair of sublethal damage by normal cells compared to malignant cells, greater reassortment of tumor cells into radiosensitive phases of the cell cycle, or reoxygenation of tumor hypoxia, and thus increased radiosensitivity. ${ }^{23}$

SRS is most effective for small, round tumor beds, because large $(>2.5 \mathrm{~cm})$ or irregularly shaped cavities may reduce treatment conformality. ${ }^{9-11,21,24,25}$ SRS to large volumes also requires irradiation of additional brain paren- chyma, increasing RN risk or necessitating dose reduction, and thus decreasing efficacy. When lesions $>1.5 \mathrm{~cm}$ are managed with SRS, RN rates of 38\% have been reported. ${ }^{21,24,26}$ Ebner et al. evaluated SRS for 343 patients, and reported 1-year LC of $68 \%$ among patients with large BM $(>3 \mathrm{~cm})$, compared to $86 \%(\mathrm{p}<0.001)$ for those with smaller lesions. ${ }^{27}$ Hartford et al. demonstrated 1-year LC of $89.1 \%$ in small tumors, versus $80.7 \%$ in large tumors. ${ }^{9}$ Ling et al. evaluated SRS specifically for large tumors $(>3$ $\mathrm{cm}$ ) and reported 72\% 1-year LC. ${ }^{11}$ Similarly, Jagannathan et al. reported increased LR of $27 \%$ in tumors with BM volume $>15 \mathrm{~cm}^{3}$, compared to $0 \%$ in tumors with smaller volumes. ${ }^{10}$ In the postoperative setting, several studies have also supported improved LC for smaller tumors. ${ }^{8,9,21}$ We did not observe this trend; we found no association between preoperative tumor size or volume and LC in our population.

SRS necessitates a delay between resection and initiation of RT, allowing for healing, cavity stabilization, and treatment planning, but also permits tumor repopulation. ${ }^{20}$ In the case of Cs-131 brachytherapy, radiation delivery begins immediately in the operating room. Indeed, in our data we observed a statistically significant increase in risk of LR with longer intervals between surgery and SRS. There is evidence that the surgical cavity experiences significant changes in size following resection. If these changes occur during the time required for SRS planning, inaccuracies in dosimetry may result. ${ }^{28,29}$ Because brachytherapy seeds are placed within the resection cavity, dose delivery is not subject to these inaccuracies. Unlike brachytherapy, adjuvant SRS requires several additional visits for imaging, treatment planning, and RT delivery. These additional steps may be burdensome, especially for patients with poor performance status, and may decrease compliance. Additionally, a recent study found that Cs-131 brachytherapy is more cost-effective than SRS, with direct hospital costs totaling $\$ 19,271$ for resection plus Cs-131 implantation, versus $\$ 44,219$ for resection plus SRS. ${ }^{30}$

Cs-131 brachytherapy targets a localized area along the perimeter of the cavity, and has a steep dose falloff, sparing surrounding parenchyma. In contrast, SRS is administered to the empty cavity, often with a small margin, and has a more gradual dose falloff, increasing radiation of normal tissues. This is consistent with our finding of decreased RN rates with Cs-131. Given the low overall incidence of RN in our study population, this difference did not reach statistical significance. In a phase I/II study of 24 patients with BM managed with postoperative Cs-131 brachytherapy, Wernicke et al. reported no RN at a median follow-up of 19 months. ${ }^{15}$ Xia et al. retrospectively evaluated 9 patients who had received surgery and Cs-131 brachytherapy, and did not encounter RN, with a median follow-up of 9 months. ${ }^{31}$ Similarly, a prospective study of patients with BM managed with resection and adjuvant Cs131 implantation reported no RN. ${ }^{32}$

We did not observe a significant difference in rates of RR, DR, or OS between patients managed with Cs-131 and SRS. This was as anticipated; we would not expect focal management of a single BM to influence distant control of intracranial disease or patient survival. We did observe a significantly longer DRFS in the Cs-131 group compared 
to the SRS group. This may relate to a delay in initiation or continuation of systemic therapy in patients treated with SRS, due to the time required to plan and deliver adjuvant RT. However, this explanation is speculative, because we did not evaluate the time to subsequent systemic therapy in our cohorts. Alternatively, this result could be attributable to some underlying difference in the cohorts not accounted for in our matching process, although as discussed, the groups did not differ significantly in any baseline characteristic evaluated.

Although there are several advantages of adjuvant intracavitary brachytherapy, there are also shortcomings to this modality. Brachytherapy seed placement confers increased radiation exposure to medical personnel compared to SRS. However, Cs-131 brachytherapy is associated with safe levels of exposure for both providers and family. ${ }^{33}$ Brachytherapy implant dosimetry is verified by CT imaging 24 to 48 hours postoperatively. There is the potential that an inadequate dose coverage is discovered, necessitating further treatment. Additionally, seed migration and resultant exposure of other tissues to radiation is possible. SRS allows radiation delivery to any location visible on MRI, whereas brachytherapy implantation is limited to areas that are visible intraoperatively.

The most significant limitation of our study was its retrospective nature. Patients were assigned to receive brachytherapy or adjuvant SRS at the provider's discretion, but were not randomized. Every effort was made to create comparable cohorts of patients receiving the two treatment modalities. Patients were matched according to the baseline variables we believed were most predictive of clinical outcomes, including tumor maximum dimension, histology, RPA class, and ECOG performance status. As shown in Table 1, the patients did not differ significantly on other clinical variables, including sex, race, KPS score, presence of active systemic disease, tumor location, presence of additional BM, surgical complications, or prior intracranial treatments. Although the cohorts were well balanced, given the retrospective nature of this study, selection bias is not eliminated, and it is possible that other confounders that were not accounted for contributed to selection of treatment modality and resultant outcomes. This shortcoming would be best addressed with a prospective, randomized trial. Our sample sizes were small, and future analyses should include more patients to improve statistical power. However, our sample was large enough to result in significant findings. The small size of the patient populations limited the selection of matched controls, resulting in potential selection bias. Although we matched our cohorts by tumor maximum dimension, histology, RPA class, and ECOG performance status, there were insufficient subjects available to allow for matching of follow-up duration or other clinical variables. Future larger, prospective, randomized studies are warranted to definitively evaluate clinical outcomes with these two adjuvant modalities.

\section{Conclusions}

In the first direct clinical comparison of intraoperative Cs-131 brachytherapy and adjuvant SRS after BM resection, we demonstrate improved LC with Cs-131 compared to SRS. There was no significant difference in RR, DR, OS, or RN rates. These findings suggest that Cs-131 is more effective at achieving LC than SRS, without compromise of other clinical outcomes, warranting further comparison in a phase III randomized trial. Based on several radiobiological, physical, dosimetric, and practical advantages, Cs-131 brachytherapy should be considered a viable and potentially preferable postoperative alternative to SRS.

\section{References}

1. Patchell RA, Tibbs PA, Walsh JW, et al. A randomized trial of surgery in the treatment of single metastases to the brain. N Engl J Med. 1990;322(8):494-500.

2. Gaspar L, Scott C, Rotman M, et al. Recursive partitioning analysis (RPA) of prognostic factors in three Radiation Therapy Oncology Group (RTOG) brain metastases trials. Int J Radiat Oncol Biol Phys. 1997;37(4):745-751.

3. Gaspar LE, Scott C, Murray K, Curran W. Validation of the RTOG recursive partitioning analysis (RPA) classification for brain metastases. Int J Radiat Oncol Biol Phys. 2000;47(4):1001-1006.

4. Kalkanis SN, Kondziolka D, Gaspar LE, et al. The role of surgical resection in the management of newly diagnosed brain metastases: a systematic review and evidence-based clinical practice guideline. J Neurooncol. 2010;96(1):33-43.

5. Chang EL, Wefel JS, Hess KR, et al. Neurocognition in patients with brain metastases treated with radiosurgery or radiosurgery plus whole-brain irradiation: a randomised controlled trial. Lancet Oncol. 2009;10(11):1037-1044.

6. Brown PD, Ballman KV, Cerhan JH, et al. Postoperative stereotactic radiosurgery compared with whole brain radiotherapy for resected metastatic brain disease (NCCTG N107C/ CEC 3): a multicentre, randomised, controlled, phase 3 trial. Lancet Oncol. 2017;18(8):1049-1060.

7. Mahajan A, Ahmed S, McAleer MF, et al. Post-operative stereotactic radiosurgery versus observation for completely resected brain metastases: a single-centre, randomised, controlled, phase 3 trial. Lancet Oncol. 2017;18(8):1040-1048.

8. Gans JH, Raper DMS, Shah AH, et al. The role of radiosurgery to the tumor bed after resection of brain metastases. Neurosurgery. 2013;72(3):317-326.

9. Hartford AC, Paravati AJ, Spire WJ, et al. Postoperative stereotactic radiosurgery without whole-brain radiation therapy for brain metastases: potential role of preoperative tumor size. Int J Radiat Oncol Biol Phys. 2013;85(3):650-655.

10. Jagannathan J, Yen CP, Ray DK, et al. Gamma Knife radiosurgery to the surgical cavity following resection of brain metastases. J Neurosurg. 2009;111(3):431-438.

11. Ling DC, Vargo JA, Wegner RE, et al. Postoperative stereotactic radiosurgery to the resection cavity for large brain metastases: clinical outcomes, predictors of intracranial failure, and implications for optimal patient selection. Neurosurgery. 2015;76(2):150-157.

12. Mahase SS, Navrazhina K, Schwartz TH, et al. Intraoperative brachytherapy for resected brain metastases. Brachytherapy. 2019;18(3):258-270.

13. Han DY, Ma L, Braunstein S, et al. Resection cavity contraction effects in the use of radioactive sources (1-25 versus Cs-131) for intra-operative brain implants. Cureus. 2018;10(1):e2079.

14. Wernicke AG, Lazow SP, Taube S, et al. Surgical technique and clinically relevant resection cavity dynamics following implantation of Cesium-131 (Cs-131) brachytherapy in patients with brain metastases. Oper Neurosurg (Hagerstown). 2016;12(1):49-60.

15. Wernicke AG, Yondorf MZ, Peng L, et al. Phase I/II study of resection and intraoperative cesium-131 radioisotope brachy- 
therapy in patients with newly diagnosed brain metastases. $J$ Neurosurg. 2014;121(2):338-348.

16. Benedict SH, Yenice KM, Followill D, et al. Stereotactic body radiation therapy: the report of AAPM Task Group 101 [erratum in: Med Phys. 2012;39(1):563]. Med Phys. 2010;37(8):4078-4101.

17. Shaw E, Scott C, Souhami L, et al. Single dose radiosurgical treatment of recurrent previously irradiated primary brain tumors and brain metastases: final report of RTOG protocol 90-05. Int J Radiat Oncol Biol Phys. 2000;47(2):291-298.

18. Cox JD, Stetz J, Pajak TF. Toxicity criteria of the Radiation Therapy Oncology Group (RTOG) and the European Organization for Research and Treatment of Cancer (EORTC). Int $J$ Radiat Oncol Biol Phys. 1995;31(5):1341-1346.

19. Kocher M, Soffietti R, Abacioglu U, et al. Adjuvant wholebrain radiotherapy versus observation after radiosurgery or surgical resection of one to three cerebral metastases: results of the EORTC 22952-26001 study. J Clin Oncol. 2011;29(2):134-141.

20. Suwinski R, Sowa A, Rutkowski T, et al. Time factor in postoperative radiotherapy: a multivariate locoregional control analysis in 868 patients. Int J Radiat Oncol Biol Phys. 2003;56(2):399-412.

21. Marchan EM, Peterson J, Sio TT, et al. Postoperative cavity stereotactic radiosurgery for brain metastases. Front Oncol. 2018;8:342.

22. Ruge MI, Kocher M, Maarouf M, et al. Comparison of stereotactic brachytherapy ( ${ }^{125}$ iodine seeds) with stereotactic radiosurgery (LINAC) for the treatment of singular cerebral metastases. Strahlenther Onkol. 2011;187(1):7-14.

23. Hall EJ, Giaccia AJ, eds. Radiobiology for the Radiologist. 7th ed. Philadelphia, PA: Lippincott Williams \& Wilkins; 2012.

24. Baschnagel AM, Meyer KD, Chen PY, et al. Tumor volume as a predictor of survival and local control in patients with brain metastases treated with Gamma Knife surgery. $\mathrm{J} \mathrm{Neu}$ rosurg. 2013;119(5):1139-1144.

25. Linskey ME, Andrews DW, Asher AL, et al. The role of stereotactic radiosurgery in the management of patients with newly diagnosed brain metastases: a systematic review and evidence-based clinical practice guideline. J Neurooncol. 2010;96(1):45-68.

26. Choi CY, Chang SD, Gibbs IC, et al. Stereotactic radiosurgery of the postoperative resection cavity for brain metastases: prospective evaluation of target margin on tumor control. Int J Radiat Oncol Biol Phys. 2012;84(2):336-342.

27. Ebner D, Rava P, Gorovets D, et al. Stereotactic radiosurgery for large brain metastases. J Clin Neurosci. 2015;22(10):1650-1654.

28. Atalar B, Choi CY, Harsh GR IV, et al. Cavity volume dynamics after resection of brain metastases and timing of postresection cavity stereotactic radiosurgery. Neurosurgery. 2013;72(2):180-185.
29. Jarvis LA, Simmons NE, Bellerive M, et al. Tumor bed dynamics after surgical resection of brain metastases: implications for postoperative radiosurgery. Int J Radiat Oncol Biol Phys. 2012;84(4):943-948.

30. Wernicke AG, Yondorf MZ, Parashar B, et al. The costeffectiveness of surgical resection and cesium-131 intraoperative brachytherapy versus surgical resection and stereotactic radiosurgery in the treatment of metastatic brain tumors. $J$ Neurooncol. 2016;127(1):145-153.

31. Xia Y, Mashouf LA, Baker BR, et al. Outcomes of metastatic brain lesions treated with radioactive Cs-131 seeds after surgery: experience from one institution. Cureus. 2018;10(7): 3075.

32. Wernicke AG, Hirschfeld CB, Smith AW, et al. Clinical outcomes of large brain metastases treated with neurosurgical resection and intraoperative cesium-131 brachytherapy: results of a prospective trial. Int J Radiat Oncol Biol Phys. 2017;98(5):1059-1068.

33. Yondorf MZ, Schwartz TH, Boockvar JA, et al. Radiation exposure and safety precautions following $131 \mathrm{Cs}$ brachytherapy in patients with brain tumors. Health Phys. 2017;112(4):403408.

\section{Disclosures}

The authors report no conflict of interest concerning the materials or methods used in this study or the findings specified in this paper.

\section{Author Contributions}

Conception and design: Wernicke, Pannullo, Schwartz. Acquisition of data: Wernicke, Julie, Lazow, Taube, Yondorf, Sabbas. Analysis and interpretation of data: Wernicke, Julie, Lazow, Vanderbilt, Taube, Yondorf, Sabbas. Drafting the article: Wernicke, Julie, Lazow. Critically revising the article: all authors. Reviewed submitted version of manuscript: all authors. Approved the final version of the manuscript on behalf of all authors: Wernicke. Statistical analysis: Julie, Lazow, Vanderbilt. Administrative/technical/material support: Sabbas. Study supervision: Wernicke, Pannullo, Schwartz.

\section{Correspondence}

A. Gabriella Wernicke: Weill Cornell Medical College, New York, NY.gabriella.wernicke5@gmail.com. 